\title{
Self-Pulsation in Two-Section Laser with an air gap
}

\author{
Chien-Chung Lin*a, Chih-Wei Lin ${ }^{\mathrm{a}}$, Chen-Yu Chien ${ }^{\mathrm{a}}$, Hao-Chung Kuo ${ }^{\mathrm{b}}$ \\ a Institute of Photonic System, College of Photonics, National Chiao-Tung University, No.301, \\ Gaofa 3rd Rd., Guiren Dist., Tainan City 71150, Taiwan; ${ }^{b}$ Department of Photonic \& Institute of \\ Electro-Optical Engineering, National Chiao Tung University, 1001 University Road, Hsinchu \\ 30010, Taiwan
}

\begin{abstract}
This study explores the optical field distribution of $1.55 \mu \mathrm{m}$ InGaAsP distributed feedback Laser with an air gap in the middle section. The optical field distribution was analyzed by different depth and width of an air gap. From the calculation, we could observe how the gap affect the coupling of the optical field into the other cavity. The percentage of the coupling is a crucial factor to the injection-locking operation. Both effective index model and commercial software were used to predict this coupling.
\end{abstract}

Keywords: Simulation of semiconductor lasers, optical field simulation, injection-locking

\section{INTRODUCTION}

In recent years there has been a growing interest in generating tunable $\mathrm{CW}$ microwave radiation, and a variety of solutions have been demonstrated for all optical 3R regeneration, re-amplification, re-shaping and re-timing. Several technologies have been explored, and two-section DFB self-pulsation laser is one of the promising candidates because of the advantages of easily DC current control of the pulsation and the ability of generating high pulsation frequency[1-5]. Specifically, there have been interests in the development of $\mathrm{THz}$ optoelectronic sources since their electronic addressability and tunability would make them easier to operate[6]. Other important applications include the microwave generation for radio over fiber (RoF) system[7], all optical clock recovery[8], etc. In this paper, we propose to use airgap between two lasers to provide optical feedback. The variation in the gap depth and width would bring about certain differences in each section, which would introduce the beating between two lasing modes, and we will analyze the optical field distribution from $1.55 \mu \mathrm{m}$ InGaAsP Fabry-Perot multisection laser with this idea. This extra air gap is designed to bring extra feedback into laser which, with careful control, can benefit the eventual coherent microwave generation.

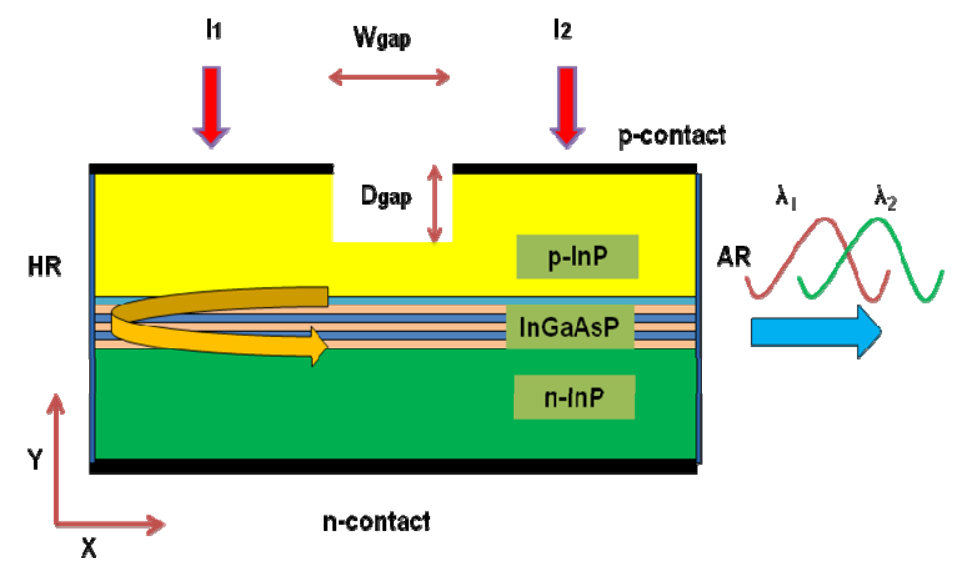

Fig. 1 The schematic diagram of our air-gap two-section distributed feedback laser.

Physics and Simulation of Optoelectronic Devices XX, edited by Bernd Witzigmann, Marek Osinski, Fritz Henneberger, Yasuhiko Arakawa, Proc. of SPIE Vol. 8255, 825523

(C) 2012 SPIE · CCC code: 0277-786X/12/\$18 · doi: 10.1117/12.908924 


\section{THEORY OF TWO-SECTION LASERS}

The operation of a two-section laser has been well studied [2]. In this paper, we put our efforts on the optical field calculation among the sections of our chip. As shown in Fig. 1, the laser chip is composed of a coupled cavity with two different coating at both facets. One side is coated with anti-reflective coating (ARC), and the other side is highreflective coating (HRC). The overall chip length is about $620 \mu \mathrm{m}$, and we design $20 \mu \mathrm{m}$ section in the middle region as our air-gap experiments. The introduction of this air gap transform the long cavity $(620 \mu \mathrm{m})$ into a coupled cavity situation: a cavity with ARC and air/semiconductor interface, an air-gap slot, and a cavity with air/semiconductor interface and HRC. All three of them are in-line together and with optical field excitation at one semiconductor section, the field will leak into the other through the air-gap slot and semiconductor underneath. Two different methods have been taken to simulate the optical field in this chip: 1. using finite element method and the cross-section of the cavity; 2. using the general waveguide theory and commercial software.

The first method was developed in Matlab ${ }^{\circledR}$ environment. The variation in the third dimension is assumed to be uniform for now. We follow the general finite element analysis criteria. The wave equation for transverse mode profile, $U$, can be written as: [9]

$$
\frac{\partial^{2} U(x, y)}{\partial x^{2}}+\frac{\partial^{2} U(x, y)}{\partial y^{2}}+k_{0}^{2}\left[n^{2}(x, y)-\bar{n}^{2}\right] U(x, y)=0
$$

We can then lay out a uniform grid which can cover overall area. After the second-order Taylor expansion of the field at the gird point, we can transform the differential equation into discretized form: [9]

$$
\begin{aligned}
& \frac{U_{j}^{i+1}-2 U_{j}^{i}+U_{j}^{i-1}}{(\Delta x)^{2}}+\frac{U_{j+1}^{i}-2 U_{j}^{i}+U_{j-1}^{i}}{(\Delta y)^{2}}+k_{0}^{2}\left[\left(n_{j}^{i}\right)^{2}-\bar{n}^{2}\right] U_{j}^{i}=0 \\
& \frac{U_{j}^{i+1}-2 U_{j}^{i}+U_{j}^{i-1}}{\left(k_{0} \Delta x\right)^{2}}+\frac{U_{j+1}^{i}-2 U_{j}^{i}+U_{j-1}^{i}}{\left(k_{0} \Delta y\right)^{2}}+\left(n_{j}^{i}\right)^{2} U_{j}^{i}=\bar{n}^{2} U_{j}^{i}
\end{aligned}
$$

, where $i$ and $j$ are the $x$-axis and $y$-axis grid indices, respectively. Once this equations are set up, the rest is the matrix eigenvalue solving, which can be easily finished in Matlab ${ }^{\circledR}$. When compared to ordinary "cold" cavity case, there are some differences: we create a refractive index difference to reflect the fact that the current injection changes the refractive index of the material.

The second method was done in the LASTIP ${ }^{\circledR}$ environment. Their theory can be traced back to the general solution of the axial field intensity. For the detailed 2D E-field distribution, we need to use a more elaborated way to simulate this problem. The basic equations used to describe the semiconductor device behavior are Poisson's equation and the current continuity equations from Maxwell's equations [10]:

$$
\begin{aligned}
& -\nabla\left(\frac{\varepsilon_{0} \varepsilon_{d c}}{q} \times \nabla V\right)=-n+p+N_{D}\left(1-f_{D}\right)-N_{A} f_{A}+\sum_{j} N_{t j}\left(\delta_{j}-f_{t j}\right) \\
& \nabla J_{n}-\sum_{j} R_{n}^{t j}-R_{s p}-R_{s t}-R_{a u}=\frac{\partial n}{\partial t}+N_{D} \frac{\partial f_{D}}{\partial t} \\
& \nabla J_{p}+\sum_{j} R_{p}^{t j}+R_{s p}+R_{s t}+R_{a u}=-\frac{\partial p}{\partial t}+N_{A} \frac{\partial f_{A}}{\partial t}
\end{aligned}
$$

, where $\mathrm{V}$ is electrical potential, $\mathrm{n}$ and $\mathrm{p}$ are electron concentration and hole concentration, $\mathrm{N}_{\mathrm{D}}$ and $\mathrm{N}_{\mathrm{A}}$ are doping of shallow donors(D) and shallow acceptors(A), $f_{D}$ and $f_{A}$ are occupancy of donor (D) and acceptor(A) levels, $N_{t j}$ is density of $j$ th deep trap, $f_{t j}$ is occupancy of the $j$ th deep trap level, $J_{n}$ and $J_{p}$ are current flux densities, $R_{n t j}$ and $R_{p t j}$ are electron and hole recombination rate for quantum well, $R_{s p}$ is spontaneous recombination rate, $R_{s t}$ is stimulated recombination 
rate, $R_{a u}$ is auger recombination rate. By solving the above equation sets, we could calculate more precisely the field distribution within the cavity.

\section{SIMULATION RESULTS}

When both sections are powered, there will be an optical feedback light at the HR side traveling through the z-direction cavity. The feedback wavelength is detuning with the original wavelength. The feedback section at HR side becomes a master laser and the other side becomes a slave laser. An air gap is located in the middle section with adjustable width and depth. There would be some optical injection locking phenomenon in the structure. Therefore, we expect that there would be some modification of dynamic characteristics of the laser, such as relative intensity noise(RIN), chirp frequency under these conditions.

\subsection{Optical Field in the Composite Cavity}

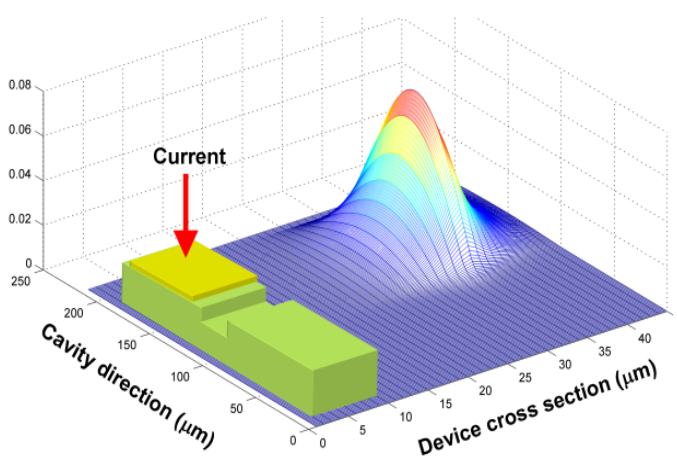

(a)

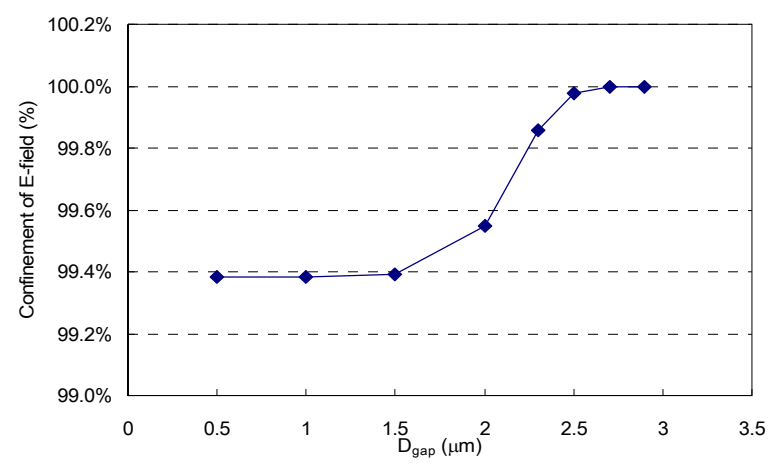

(b)

Figure 2. (a) 2-dimensional simulation of pumped dual-section cavity; (b) Calculation of confinement of E-field in the quantum well axial direction.

Fig. 2(a) shows the two dimensional field distribution. Once we obtain the optical field in the pumped cavity, we could integrate the relative intensity over each section and compare their ratio. The percentage of the field intensity in the pumped cavity is plotted in Fig. 2(b), and we can find out a sharp increase of confinement when the $\mathrm{D}_{\text {gap }}$ increases more than $2 \mu \mathrm{m}$. However, we could see that the optical confinement of the fundamental mode of this setup is quite good, even with the $0.5 \mathrm{um}$, we only have about $0.6 \%$ of the light field leaking into the next cavity. So the finite element simulation can only give us an idea on how fast the confinement rises, but not the absolute value of the actual confinement.

On the Lastip ${ }^{\circledR}$ calculation, we started the simulation under $\mathrm{R}_{1}=\mathrm{R}_{2}=0.32$ and $\mathrm{I}_{2}$ off. We focus now on the wave intensity distribution with an air gap of different depths and widths. Figure 3(a) shows the E-field distribution without any gap. Figure 3(b) shows that the wave intensity is re-distributed when the depth of the air gap is increased to $5 \mu \mathrm{m}$, the field is hardly penetrated into the right section. We calculated the wave intensity distribution ratio of the pumped cavity versus the overall field intensity shown at the Table 1 . When there is no depth on the chip, the left field intensity is about $50.3 \%$ at width of gap is $2 \mu \mathrm{m}$ and is $55.4 \%$ at the width of the air gap is $5 \mu \mathrm{m}$. There is little difference of intensity ratio between the two cases. However, once we start increase $\mathrm{D}_{\text {gap }}$, and widen $\mathrm{W}_{\text {gap }}$, the obvious partition of field intensity can be observed. The detailed 2 dimensional calculation is summarized in table 1. As we could see, the influences of the air gap is profound. Most of the excited E-field is confined in the pumped region, however, some of them will leak into the other un-pumped (or cold) cavity. This leakage is the source of interference of the other section of laser and usually we don't know, to what extent, this leakage will disturbing the operation of the other laser unless we can quantify it. Using this method, we can estimate the possible feedback or coupling between multiple sections of semiconductor lasers. 


\subsection{L-I Simulation and Measured Results}

When we put a air slot in the middle section of the two-section laser, the basic performances such as laser power, current distribution or leakage current, which could be influenced to what extent by different $\mathrm{D}_{\text {gap.. }}$ Therefore, we have to do some simulation and measurement about the basic performances after the focus ion beams process.

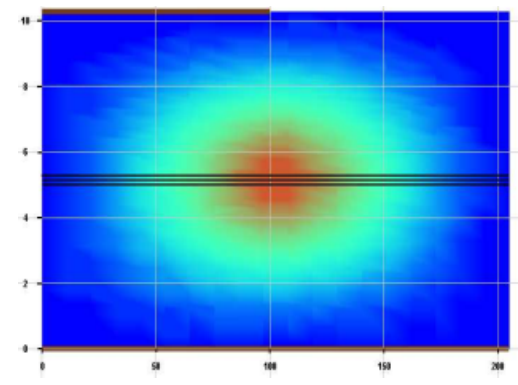

(a)

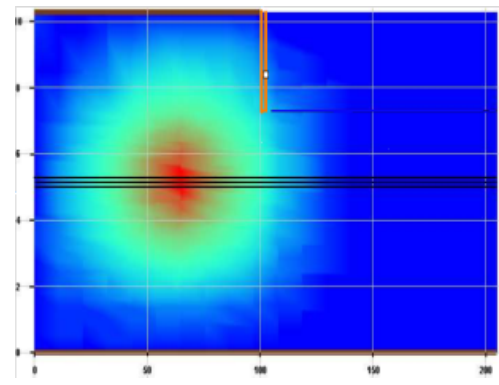

(b)

Figure 3. (a) Field distribution: $\mathrm{W}_{\text {gap }}=5 \mu \mathrm{m}$, but with no air gap; (b) Field distribution on $\mathrm{W}_{\text {gap }}=5 \mu \mathrm{m}, \mathrm{D}_{\text {gap }}=5 \mu \mathrm{m}$

Table 1. Field intensity ratio on the pumped cavity

\begin{tabular}{|c|c|c|c|}
\hline $\mathrm{W}_{\text {gap }}$ & $\mathrm{D}_{\text {gap }}=0 \mu \mathrm{m}$ & $\mathrm{D}_{\text {gap }}=3 \mu \mathrm{m}$ & $\mathrm{D}_{\text {gap }}=5 \mu \mathrm{m}$ \\
\hline $2 \mu \mathrm{m}$ & $50.3 \%$ & $90.1 \%$ & $90.4 \%$ \\
\hline $3 \mu \mathrm{m}$ & $49.7 \%$ & $90.8 \%$ & $93 \%$ \\
\hline $5 \mu \mathrm{m}$ & $55.4 \%$ & $93.4 \%$ & $94 \%$ \\
\hline
\end{tabular}

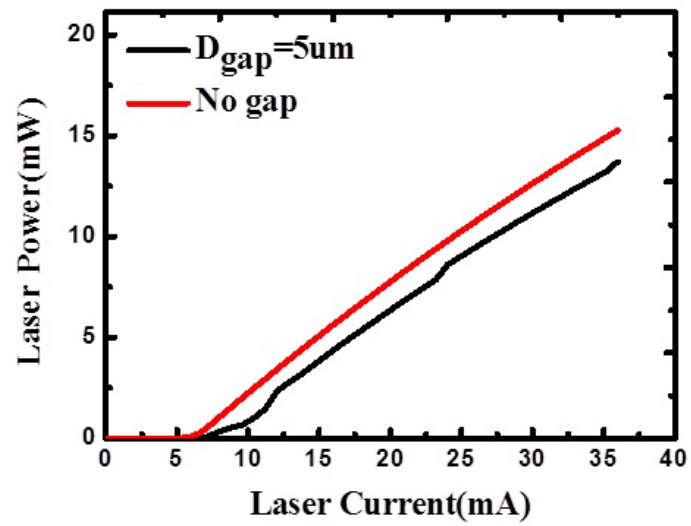

(a)

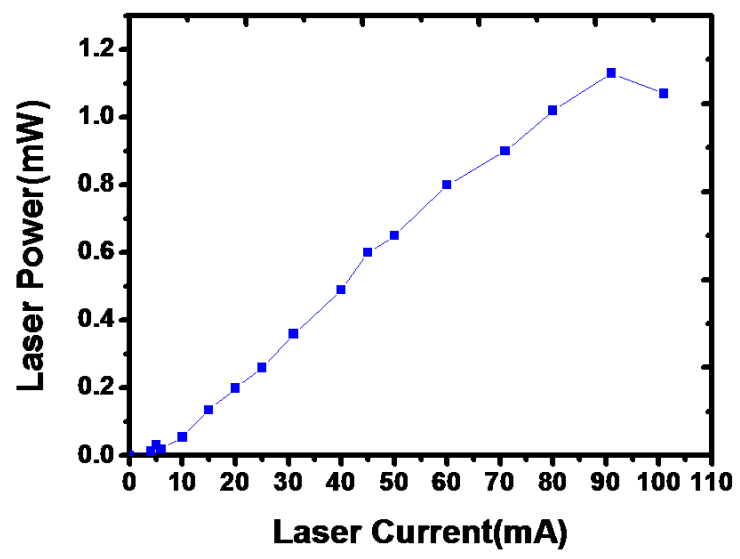

(b)

Figure 4. L-I curve (a) simulation results with air gap $\mathrm{D}_{\text {gap }}=5 \mathrm{um}$ and with no gap (b) measurement result with $\mathrm{D}_{\text {gap }}=5 \mathrm{um}$

The figure 3.3.1(a) shows the L-I simulation result of un-FIB laser, which is normal average performance on the twosection laser. The figure 3.3.1(b) shows the L-I measurement result under different bias current. In addition, the threshold current is matches, while the power is decrease at $91 \mathrm{~mA}$ due to large bias current which leads to the spatial hole burning.

If we etch the laser to $D_{\text {gap }}=5 \mathrm{um}$, the threshold current could increase and the laser power decrease a little. Hower,etching to the active layer, we can see not only the threshold current will increase more but also the laser power will fall down sharply. We could have the most moderate $\mathrm{D}_{\text {gap }}$ to etch the two section laser. 


\subsection{Leakage current}

Our monolithic two-section laser is not composed of two independent lasers. The two sections have a common laser grating and an optical cavity originally. After the FIB etching process, the monolithic laser has two asymmetric laser cavities, but it still have common active layers. So, the other characteristic what we want to know is the laser current distribution after FIB process.

Because the pumped cavity current could leak through the cold cavity, it might give rise to some influence on cold cavity. From the Figure 5(a), we can see that some leakage current inject through the active layer on the cold cavity with an air gap. However, Figure 5(b) shows that if we cut through the active layer, the pumped cavity current will almost not leak out to the active layer of the cold cavity.

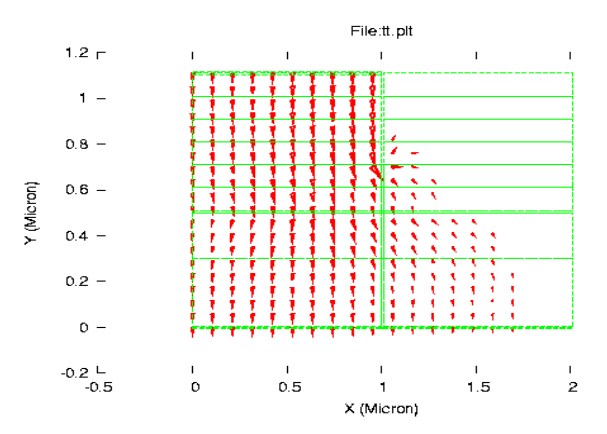

(a)
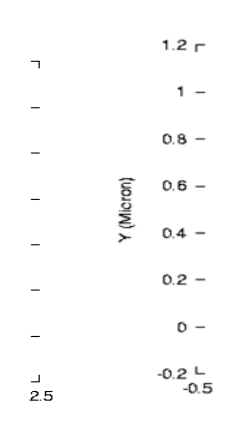

Figure 5. Current distribution with different depths of the air gap (a)

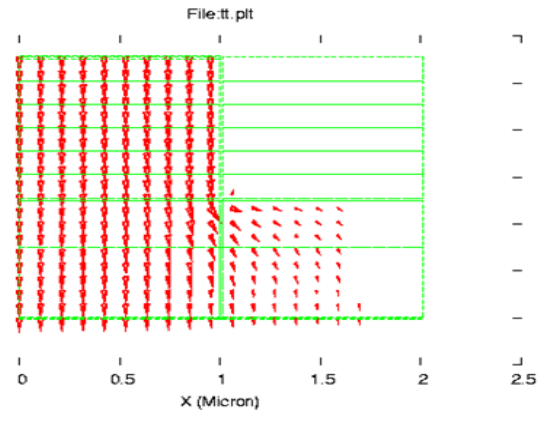

(b)

\subsection{Injection-locking Condition Calculation}

The injection-locking calculation is also necessary for us to estimate the possible operating range of the device. The principles can be found in reference 10 for details. We use the edge-emitting laser gain coefficient, which is about 100 times larger than that of VCSEL's. The linewidth enhancement factor can be between 2 to 5 according to the past research[11]. Putting all these parameters together, we can have a rough estimate on how our microwave generation is going to evolve when we pump the master laser. Fig. 6 shows the result of the injection-locking range calculation. From these calculation, we can see that the smaller the linewidth enhancement factor is, the larger the injection-locking bandwidth will be.
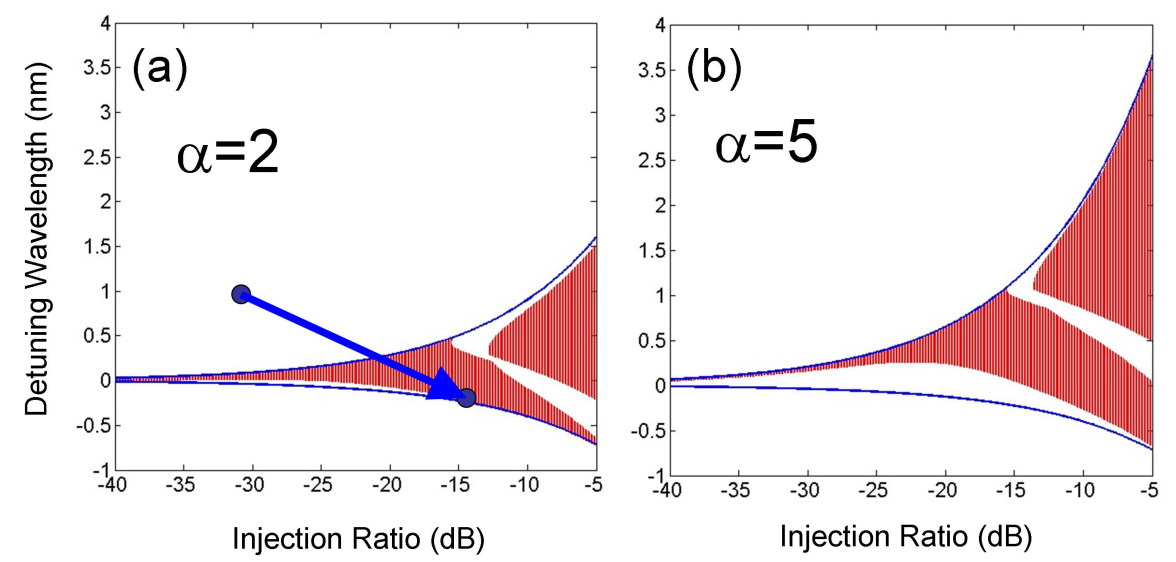

Figure 6. The injection-locking bandwidth vs. injection ratio for different linewidth enhancement factor (2 and 5). The arrow shows the possible trajectory of our device when the master laser is pumping the slave laser. The red color area indicates the stable locking region, and the white color area is the unstable region. 


\section{CONCLUSIONS}

In conclusion, we demonstrate a theoretical calculation based on different depths and widths of the air gap to change the optical field distribution. We can thus understand the ratio the optical field from the other adjacent cavity that couples into the main cavity. With the knowledge of optical field distribution, we could further calculate the dynamic characteristics of microwave signal generation such as relative intensity noise(RIN), resonance frequency ,chirp frequency. The possible influences on the LI performance due to introduction of air-gap is also calculated and measured. The air-gap in the two-section lasers is important feature, which can affect the coupling between the two cavities and also the overall power.

\section{ACKNOWELDGEMENT}

The authors would like to thank the financial support of National Council of Science of Taiwan, R.O.C. through the grant number: NSC 99-2221-E-009-052-MY3

\section{REFERENCES}

[1] José Capmany \& Dalma Novak, " Microwave photonics combines two worlds, " Nature Photonics, 1, 319 - 330 (2007).

[2] Christina Lim, Dalma Novak, Ampalavanapillai Nirmalathas, and Graham H. Smith, "Dispersion-Induced Power Penalties in Millimeter-Wave Signal Transmission Using Multisection DBR Semiconductor Laser, “ IEEE Transactions On Microwave Theory And Techniques, 49(2), 288-296 (2001)

[3] Sze-Chun Chan, Sheng-Kwang Hwang, and Jia-Ming Liu, "Period-one oscillation for photonic microwave transmission using an optically injected semiconductor laser," Opt. Express, 15, 14921-14935 (2007).

[4] R.K. Price, V.B. Verma, K.E. Tobin, K.E. Elarde, J.J. Coleman, "Y-Branch Surface-Etched Distributed Bragg Reflector Lasers at $850 \mathrm{~nm}$ for Optical Heterodyning, "IEEE Photonics Technology Letters, 19 (20), 1610-1612 (2007).

[5] Ning Hua Zhu, Member, IEEE, Wei Li, Li Xian Wang, Shuo Fu Chen, Jian Hong Ke, Ya Li Zhang, Ji Min Wen, Yu Liu, Xin Wang, Hai Qing Yan, and Liang Xie, "Study on Frequency Coherence Properties of Light Beams, "IEEE Journal Of Quantum Electronics, 45(5), 514-522, (2009).

[6] A. Klehr, J. Fricke, A. Knauer, G. Erbert, M. Walther, R. Wilk, M. Mikulics, and M. Koch, "High-power monolithic two-mode DFB laser diodes for the generation of THz radiation," IEEE J. Sel. Top. Quantum Electron. 14(2), 289-294 (2008).

[7] Chun-Ting Lin, J. Chen, Peng-Chun Peng, Cheng-Feng Peng, Wei-Ren Peng, Bi-Shiou Chiou, S. Chi, "Hybrid Optical Access Network Integrating Fiber-to-the-Home and Radio-Over-Fiber System, " IEEE Photonic Technology Letters, 19, 610-612 (2007).

[8] C. Bornholdt, B. Sartorius, S. Schelhase, M. Mohrle, and S. Bauer, "Self-pulsating DFB laser for all-optical clock recovery at $40 \mathrm{Gbit} / \mathrm{s}$, " Electron. Lett. 36, 327 (2000)

[9] L. A. Coldren, S. W. Corzine, "Diode Lasers and Photonic Integrated Circuits, " Wiley-Interscience; New York, (1995)

[10] APSYS User's Manual Version 2003.4 Edition 1, p. 38, Chapter 4.

[11]P. Thijs, L. F. Tiemeijer, P. I. Kuindersma, J. J. M. Binsma, and T. Van Dongen, " High-Performance 1.5mm Wavelength InGaAs-InGaAsP Strained Quantum Well Lasers and Amplifiers", IEEE Journal of Quantum Electronics, 27(6), 1426 (1991). 